\title{
Real-Time Receding-Horizon Control Algorithm for Nonlinear Systems
}

\author{
Toshiyuki OHTsukA* and Hironori A. FuJII**
}

\begin{abstract}
A real-time optimization technique is proposed for optimal state feedback control of general nonlinear systems. A state feedback control law is determined so that a receding-horizon performance index is minimized. Computation of the control input is equivalent to solving a family of optimal control problems with a varying initial state in the real time, for which the stabilized continuation method is applicable. Application of the stabilized continuation method results in a real-time solution technique that does not involve any approximation nor iterative algorithm in principle. The proposed solution technique successfully implemented in a control experiment of a 2 -wheeled mobile robot that is nonlinear and nonholonomic. Equilibrium points of the closed-loop system are also analyzed.
\end{abstract}

Key words: real-time optimization, receding-horizon control, nonlinear control, continuation method, nonholonomic system

\section{Introduction}

In classical optimal control theories, optimal feedback laws are obtained by solving the Hamilton-Jacobi-Bellman equation $^{1)}(\mathrm{HJBE})$ for optimal control problems. The HJBE is a nonlinear partial differential equation including the time derivative for a finite horizon problem, and reduces to a nonlinear partial differential equation without the time derivative for an infinite horizon problem ${ }^{2}$. The HJBE can not be solved analytically in general. Infinite horizon regulators do not depend on time explicitly with a guarantee on the closed-loop stability under some conditions, and have certain robustness properties ${ }^{3)}$, which are preferable features for feedback control. In contrast to infinite horizon regulators, optimal feedback laws depend on time explicitly, and the closed-loop stability is not guaranteed for finite horizon problems in general.

However, a time-independent feedback law is obtained and the closed-loop is stabilized by the feedback law, if an optimal control problem has a moving terminal time and the terminal state is constrained to be $z^{2}{ }^{4), 5}$. This concept of a moving terminal time is called the receding horizon or moving horizon, and is applied originally to linear systems ${ }^{6)-8}$. Chen and Shaw $^{4)}$ and Mayne and Michalska ${ }^{5}$ apply the receding horizon control to general

\footnotetext{
* Institute of Engineering Mechanics, University of Tsukuba, Tsukuba

** Department of Aerospace Engineering, Tokyo Metropolitan Institute of Technology, Hino

(Received December 16, 1996)

(Revised August 18, 1997)
}

nonlinear systems, and show that the closed-loop is asymptotically stable if the terminal state is constrained to be zero. The case with a relaxed terminal constraint is also considered in Ref. 9. The receding horizon control for nonlinear systems can be regarded as a nonlinear version of the model predictive control ${ }^{10)}$ in the sense that the control performance is predicted and optimized over a finite interval in the future.

When a control law is designed as the receding horizon control, a two-point boundary-value problem (TPBVP) needs to be solved in order to determine the control input, and the solution technique is a critical issue in the implementation of the feedback law. Category of solution techniques are discussed and interpolation techniques are considered in Ref. 11. In the present paper, we propose a real-time solution technique for the nonlinear receding horizon control without terminal constraints. The solution technique presented in this paper does not involve any approximation nor iterative algorithms in contrast to realtime optimization techniques in Refs. 12-14 that discretize the problem and employ iterative approximation. It is shown that the receding horizon control is equivalent to solving a family of optimal control problems with a vary ing initial state, and the stabilized continuation method ${ }^{15}$ is applied to solve it in the real time. The unknown costate in the optimal control problem is traced by varying the initial state and the terminal time starting from a known solution. A stabilizing term is introduced to correct the error efficiently in tracing the optimal solution.

The proposed solution technique is applied to a control experiment of a 2 -wheeled mobile robot that is nonlinear 
and nonholonomic. In spite of modeling error, the proposed solution technique generates accurate solutions, and the experimental model is controlled successfully. The results of the experiment validate that the proposed technique is suitable to practical use. Equilibrium points of the closed-loop system are also analyzed.

\section{Problem Formulation}

The dynamical system treated here is expressed in the following differential equation :

$$
\frac{d x(t)}{d t}=f[x(t), u(t)]
$$

where $x$ denotes the state vector and $u$ the control input vector. A time-variant system can also be expressed in the above form if the time is regarded as a state of the system. We are interested in the determination of a control law that depends only on the state of the system. Usual optimal control problems are not suitable for the present purpose, since the optimal control input depends on time explicitly in general. In this paper, the control law is determined by minimizing a receding-horizon performance index :

$$
J=\varphi[x(t+T)]+\int_{t}^{t+T} L[x(\eta), u(\eta)] d \eta
$$

Any terminal constraints are not imposed on $x(t+T)$ in the present formulation. Note that the performance index evaluates the control performance from the present time $t$ until the finite future $t+\underset{T}{T}$. The optimal control input that minimizes the above performance index is a function of the time over the interval $[t, t+T]$, and also depends on the present state $x(t)$. However, only the optimal control at the present time $t$ is used as the actual control input to the system. Since both of the system dynamics and the performance index do not depend on time explicitly, the actual control input depends only on the present state $x(t)$. That is to say, the control law is determined as a state feedback. Since the performance index is evaluated over a finite interval, the value of the performance index is finite and the optimal control problem has a solution even for a system that is not stabilizable. Therefore the receding-horizon state feedback control is determined even if the system is not stabilizable, while a performance index may not be bounded and there may not be any solutions in an infinite-horizon regulator problem for a system that is not stabilizable. The receding horizon control can be designed for a broader class of systems in comparison with the infinite horizon regulator.

By introducing a new time variable $\tau$, the receding horizon control problem can be converted to a family of optimal control problems parameterized by the actual time $t$ as follows :

Minimize : $J=\varphi\left[x^{*}(T, t)\right]+\int_{0}^{T} L\left(x^{*}(\tau, t), u^{*}(\tau, t)\right] d \tau$

Subject to : $\frac{\partial x^{*}(\tau, t)}{\partial \tau}=f\left[x^{*}(\tau, t), u^{*}(\tau, t)\right]$;

$x^{*}(0, t)=x(t)$

with the actual control input $u(t)$ given by :

$$
u(t)=u^{*}(0, t)
$$

where $(\quad)$ denotes a variable in the parameterized optimal control problem in order to distinguish it from its correspondence in the actual system. The optimal control problem has the initial state $x^{*}(0, t)=x(t)$ that varies with respect to the actual time $t$, and is solved along the fictitious time $\tau$. The terminal time $T$ also varies in general, as is explained later. Note that the converted optimal control problem is a standard Bolza type problem for a fixed pair of $t$ and $T$, and the actual control input is given by the initial value of the optimal control input minimizing the performance index. The first order necessary conditions for the optimal solution are obtained readily as a TPBVP by the calculus of variations as: ${ }^{1)}$

$$
\begin{aligned}
& \frac{\partial x^{*}(\tau, t)}{\partial \tau}=H_{\lambda}^{T} ; x^{*}(0, t)=x(t) \\
& \frac{\partial \lambda^{*}(\tau, t)}{\partial \tau}=-H_{x}^{T} ; \lambda^{*}(T, t)=\varphi_{x}^{T}\left[x^{*}(T, t)\right] \\
& H_{u}=0
\end{aligned}
$$

where $H$ denotes the Hamiltonian defined as :

$$
H=L+\lambda^{* T} f
$$

and $H_{x}$ denote the partial derivative of $H$ with respect to $x^{*}$ and so on. The parameterized initial costate $\lambda^{*}(0, t)$ is denoted by $\lambda(t)$ corresponding to the initial state $x^{*}(0, t)=$ $x(t)$.

Since the terminal state $x^{*}(T, t)$ and the terminal costate $\lambda^{*}(T, t)$ are determined by Eqs. (6)-( 8$)$ from the given initial state $x^{*}(0, t)=x(t)$ and the unknown initial costate $\lambda^{*}(0, t)=\lambda(t)$, the TPBVP can be regarded as a nonlinear equation with respect to the initial costate as:

$$
F[\lambda(t), x(t), T]=\lambda^{*}(T, t)-\varphi_{x}^{T}\left[x^{*}(T, t)\right]=0
$$

The initial costate $\lambda^{*}(0, t)=\lambda(t)$ is determined implicitly at each time $t$ by Eq. (10) from the initial state $x^{*}(0, t)=$ $x(t)$, and the terminal time $T$.

\section{Real-Time Solution by Stabilized Continuation Method}

In order to solve the optimal control problem in the real time with moderate computational load and data storage, this paper employs a non-iterative solution technique, the stabilized continuation method. The problem is converted 
to an initial-value problem of an ordinary differential equation that can be solved numerically without recourse to iterative methods. A general discussion of the stabilized continuation method is given in Ref. 15 for solving optimal control problems with general boundary constraints and nondifferential equality constraints.

The continuation methods are usually applied to problems that do not have to be solved in the real time. However, the receding horizon control problem requires a real-time algorithm in the sense that the algorithm have to proceed with the time in the real world. Therefore this paper proposes an algorithm that traces the solution varying with the actual time $t$, which contrasts with the usual continuation methods that trace solutions with respect to fictitious parameters.

Since the nonlinear equation (10) has to be satisfied at any time $t, d F / d t=0$ holds along the trajectory of the closed-loop system of the receding horizon control. An ordinary differential equation for $\lambda(t)=\lambda^{*}(0, t)$ is obtained from $d F / d t=0$ with respect to the actual time $t$, and can be integrated with numerical algorithms without recourse to any iterative approximation methods if an initial condition is given. However, numerical error in the solution may accumulates through the numerical integration in practice, and some correcting techniques are necessary in the numerical algorithm. While the conventional continuation methods utilize such an iterative algorithm as the Newton method in order to correct the error ${ }^{16), 17}$, this paper modifies the continuation method so that the error attenuates as the integration proceeds as follows:

$$
\frac{d F}{d t}=v
$$

where $v$ denotes the stabilizing input to attenuate the error. The stabilizing input $v$ may be chosen, for example, as :

$$
v=A_{S} F
$$

where $A_{S}$ denotes a stable matrix, i. e., every eigenvalue of $A_{s}$ has a negative real part. This choice of $v$ results in the attenuation of the error as :

$$
F=F_{0} e^{A_{S} t} \rightarrow 0 \quad(t \rightarrow \infty)
$$

where $F_{0}$ denotes the initial value of $F$. A stabilizing input for the continuation method is introduced in Ref. 18, and more broad class of the stabilizing inputs are introduced and discussed in Ref. 15. Although use of the stabilizing term allows nonzero $F_{0}$ in principle, $F_{0}$ should be set zero since initial error in the optimal solution may cause a fatal control action.

The differential equation for $\lambda(t)=\lambda^{*}(0, t)$ is obtained from Eq. (11) as follows :

$$
\frac{d \lambda(t)}{d t}=\left(\frac{\partial F}{\partial \lambda}\right)^{-1}\left[v-\frac{\partial F}{\partial x} \frac{d x(t)}{d t}-\frac{\partial F}{\partial T} \frac{\partial T(t)}{d t}\right]
$$

where the symbols $\partial F / \partial \lambda$ and $\partial F / \partial x$ denote the partial derivatives of $F$ with respect to $\lambda(t)=\lambda^{*}(0, t)$ and $x(t)=$ $x^{*}(0, t)$, respectively. The terminal time $T$ is regarded as a smooth function of the time $t$ such that $T(0)=0$ and $T(t) \rightarrow T_{f}(t \rightarrow \infty)$, where $T_{f}$ denotes the terminal time prescribed by a designer. The terminal time $T(t)$ is increased from zero gradually so that the optimal control problem has a trivial solution at $t=0$. Since $\lambda(0)=\lambda^{*}[T(0)$ $0]$ and $x(0)=x^{*}[T(0), 0]$ hold and Eq. (10) has to be satisfied, the initial condition at $t=0$ for Eq.(14) is given by:

$$
\lambda(0)=\varphi_{x}^{T}[x(0)]
$$

When the terminal time depends on time, the control law also depends on time even for a time-invariant system. However, the optimality condition Eq.(10) is satisfied throughout the control process, and the control law converges to a time-invariant control law as the terminal time $T(t)$ converges to the constant value $T_{f}$.

In order to evaluate the partial derivatives in Eq. (14), we consider the variation in the optimal solution caused by the infinitesimal variation in the time $t$. It is straightforward to show that the variations in the state and costate are governed by the following linear differential equation $^{1)}$ :

$$
\frac{\partial}{\partial \tau}\left[\begin{array}{l}
\delta x^{*}(\tau, t) \\
\delta \lambda^{*}(\tau, t)
\end{array}\right]=\left[\begin{array}{cc}
A(\tau, t) & -B(\tau, t) \\
-C(\tau, t) & -A^{T}(\tau, t)
\end{array}\right]\left[\begin{array}{l}
\delta x^{*}(\tau, t) \\
\delta \lambda^{*}(\tau, t)
\end{array}\right]
$$

where $A, B$ and $C$ are evaluated along the optimal solution as follows:

$$
\begin{aligned}
& A=f_{x}-f_{u} H_{u u}^{-1} H_{u x} \\
& B=f_{u} H_{u u}^{-1} f_{u}^{T} \\
& C=H_{x x}-H_{x u} H_{u u}^{-1} H_{u x}
\end{aligned}
$$

The variations $\delta x^{*}(\tau, t)$ and $\delta \lambda^{*}(\tau, t)$ are expressed in terms of the transition matrix of Eq. (16) as follows:

$$
\left[\begin{array}{l}
\delta x^{*}(\tau, t) \\
\delta \lambda^{*}(\tau, t)
\end{array}\right]=\left[\begin{array}{ll}
\Phi_{11}(\tau, t) & \Phi_{12}(\tau, t) \\
\Phi_{21}(\tau, t) & \Phi_{22}(\tau, t)
\end{array}\right]\left[\begin{array}{l}
\delta x^{*}(0, t) \\
\delta \lambda^{*}(0, t)
\end{array}\right]
$$

The transition matrix is parameterized by the actual time $t$ as well as the variations. The transition matrix is the solution of the following differential equation:

$$
\begin{aligned}
& \frac{\partial}{\partial \tau} {\left[\begin{array}{cc}
\Phi_{11}(\tau, t) & \Phi_{12}(\tau, t) \\
\Phi_{21}(\tau, t) & \Phi_{22}(\tau, t)
\end{array}\right] } \\
& \quad=\left[\begin{array}{cc}
A(\tau, t) & -B(\tau, t) \\
-C(\tau, t) & -A^{T}(\tau, t)
\end{array}\right]\left[\begin{array}{ll}
\Phi_{11}(\tau, t) & \Phi_{12}(\tau, t) \\
\Phi_{21}(\tau, t) & \Phi_{22}(\tau, t)
\end{array}\right]
\end{aligned}
$$

with the initial condition:

$$
\left[\begin{array}{ll}
\Phi_{11}(0, t) & \Phi_{12}(0, t) \\
\Phi_{21}(0, t) & \Phi_{22}(0, t)
\end{array}\right]=\left[\begin{array}{ll}
I & 0 \\
0 & I
\end{array}\right]
$$

The variation in the function $F$ is expressed as: 


$$
\begin{aligned}
d F= & \delta \lambda^{*}(T, t)-\left.H_{x}^{T}\right|_{\tau=T} d T \\
- & \left.\varphi_{x x}\right|_{\tau=\tau}\left[\delta x^{*}(T, t)+\left.f\right|_{\tau=T} d T\right] \\
= & \left.\left(\Phi_{22}-\varphi_{x x} \Phi_{12}\right)\right|_{\tau=T} \delta \lambda^{*}(0, t) \\
& +\left.\left(\Phi_{21}-\varphi_{x x} \Phi_{11}\right)\right|_{\tau=T} \delta x^{*}(0, t) \\
& -\left.\left(\varphi_{x x} f+H_{x}^{T}\right)\right|_{\tau=\tau} d T
\end{aligned}
$$

where the coefficient matrices of $\delta \lambda^{*}(0, t), \delta x^{*}(0, t)$ and $d T$ represent partial derivatives of $F$ with respect to $\lambda, x$ and $T$, respectively. In summary, the control law based on the stabilized continuation method is given by:

$$
u(t)=\arg \left\{H_{u}[x(t), \lambda(t), u(t)]=0\right\}
$$

where the state $x(t)$ is given, and the costate $\lambda(t)$ is governed by the following ordinary differential equation:

$$
\begin{aligned}
\frac{d \lambda(t)}{d t}= & \left.\left(\Phi_{22}-\varphi_{x x} \Phi_{12}\right)\right|_{\tau=T} ^{-1} \\
& \times\left[v(t)+\left.\left(\varphi_{x x} \Phi_{11}-\Phi_{21}\right)\right|_{\tau=T} \frac{d x(t)}{d t}\right. \\
& \left.+\left.\left(\varphi_{x x} f+H_{x}^{T}\right)\right|_{\tau=T} \frac{d T(t)}{d t}\right]
\end{aligned}
$$

with the initial condition Eq. (15). It should be noted that Eq. (25) is the exact differential equation for the costate if the optimality condition $F=0$ holds and the stabilizing term $v$ is zero. The differential equation. (25) can be integrated numerically without any successive approximation, which is a significant difference from the conventional real-time optimization techniques in Refs. 12-14.

The control law Eqs. (24) and (25) has to be discretized with respect to the time for implementation on a digital computer. The computation of the optimal control input involves the integration of the variational equation and solution of a linear equation associated with the inverse matrix $\left(\Phi_{22}-\varphi_{x x} \Phi_{12}\right)^{-1}$. The whole dimension of the differential equations (Euler-Lagrange equations and variational equation) to be integrated is given by $2 n(2 n+1) \cong$ $4 n^{2}$ for an $n$ dimensional system, and the number of the integration steps may be evaluated in proportion to the desired terminal time $T_{f}$. Therefore the computational cost of integration is estimated by $k_{1} n^{2} T_{f}$ for a some positive constant $k_{1}$. Since the computational cost of the linear equation is estimated by $O\left(n^{3}\right)$, the total computational cost of the control law is estimated by $k_{1} n^{2} T_{f}+k_{2} n^{3}$ where $k_{2}$ is a positive constant.

\section{Hardware Experiment of a 2-Wheeled Mobile Robot}

\section{1 Experiment Setup}

The proposed method is applied to a control experiment in order to verify that it can be implemented for real-time feedback control. The experimental model (Fig. 1) to be controlled is a 2 -wheeled mobile robot. The model is driven by two DC motors in the horizontal plane. The motors are inclined so that the edges of their shafts contact with the floor to generate thrust. The position and attitude of the experimental model are measured at the sampling interval of 0.1 [sec] by an optical position sensor that tracks positions of two LEDs on the experimental model. The positions of the LEDs are transferred to a digital computer through RS-232 C. The digital computer (CPU 386+ NDP 387, clock $16 \mathrm{MHz}$ ) processes the sensor data, and put out control signals through a D/A converter. The output voltages from the $\mathrm{D} / \mathrm{A}$ converter are put into a power booster that supplies current to the DC motors. The DC motors are modeled to rotate at velocities that are proportional to the input voltage. The actual velocities of the motors track the command velocities with a time delay of about 0.2 seconds, which is sufficiently small compared to the execution time of a few ten seconds in the present experiment and justifies the simple modeling of the motors.

\subsection{Control Design}

Under the assumption that the velocities of the two motors are given as the control input, the state variables of the experimental model are its position $\left(x_{1}, x_{2}\right)$ and its attitude angle $x_{3}$ (see Fig. 2). The mathematical model of the experimental model is expressed in the following nonlinear system :

$$
\frac{d x}{d t}=G(x) u
$$

where $x=\left[\begin{array}{lll}x_{1} & x_{2} & x_{3}\end{array}\right]^{T}$ denotes the state vector, $u=\left[\begin{array}{ll}u_{1} & u_{2}\end{array}\right]^{T}$
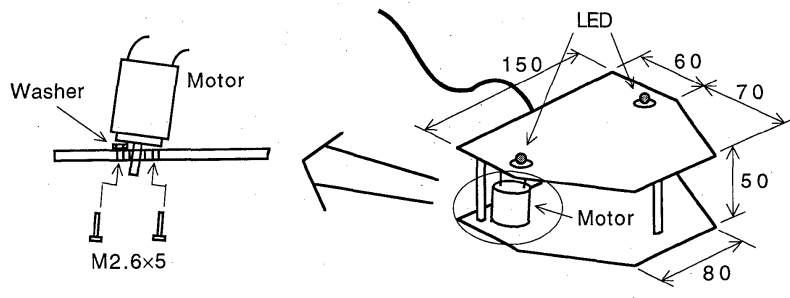

(Unit: $\mathrm{mm}$ )

Fig. 1 Experimental model.

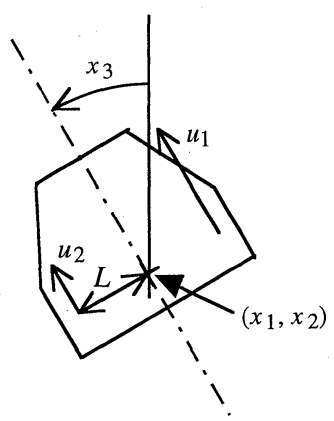

Fig. 2 2-wheeled mobile robot. 
the control input, and

$$
G(x)=\left[\begin{array}{ll}
g_{1}(x) & g_{2}(x)
\end{array}\right]=\frac{1}{2}\left[\begin{array}{cc}
-\sin x_{3} & -\sin x_{3} \\
\cos x_{3} & \cos x_{3} \\
1 / L & -1 / L
\end{array}\right]
$$

We identify the state space with the Euclidean space $\boldsymbol{R}^{3}$ in order to avoid the necessity to switch the local coordinates in the control process. Therefore the attitude angle $x_{3}$ is not restricted to the range of $0 \leq x_{3}<2 \pi$. The fundamental result of the nonlinear control theory ${ }^{19)}$ implies that the present model is controllable on the whole state space. However, the present model is nonholonomic. The theorem of Brockett ${ }^{20)}$ implies that there is no smooth state feedback law that stabilizes the origin asymptotically in the present system. Therefore an infinite-horizon regulator is hard to design for the present system. However the receding-horizon control problem can generate a smooth feedback law, though it may not result in the asymptotic stability of the closed-loop system.

The control objective in the experiment is to bring the model to the origin and to set the attitude angle zero. One of the simplest performance indexes that reflect the control objective is the following quadratic performance index :

$$
J=\frac{1}{2} x^{T}(t+T) S_{f} x(t+T)+\int_{t}^{t+T} \frac{1}{2} u^{T}(\eta) u(\eta) d \eta
$$

where

$$
S_{f}=\operatorname{diag}\left(s_{1}, s_{2}, s_{3}\right), T(t)=T_{f}\left(1-e^{-\alpha t}\right)(\alpha>0)
$$

The variable terminal time $T(t)$ satisfies $T(0)=0$, and $T(t)$ converges to the desired terminal time $T_{f}$ as the time increases. The control inputs are included in the performance index so that the optimal control has bounded magnitude, and the state vector is not included in the integrant in order to reduce the computational cost in integrating the Euler-Lagrange equations. The model is controlled by the bounded control input so as to minimize the deviation from the desired state at the time $T(t)$ away. The optimal control is obtained from the optimality condition as :

$$
u=-G^{T} \lambda
$$

where the costate $\lambda$ is computed by the stabilized continuation method. The stabilizing input is chosen as $v=-\zeta F(\zeta$ $>0$ ), and the differential equation of the costate, Eq. (25), is discretized by forward difference.

\section{3 Experimental Results}

The initial state is given in this experiment as $x_{1}=$ $0.3[\mathrm{~m}], x_{2}=0.2[\mathrm{~m}]$, and $x_{3}=\pi / 2[\mathrm{rad}]$. Weights for the terminal penalty are set as $\left(s_{1}, s_{2}, s_{3}\right)=(1,1,0.001)$. Some different situations are compared so as to clarify charac- teristics of the control law. The terminal time $T$ is set zero identically in order to show the necessity for prediction in the first case. The costate $\lambda(t)$ in this case is obtained explicitly as $\lambda(t)=S_{f} x(t)$ from the terminal condition, and the control law is given by :

$$
u=-G^{T} S_{f} x
$$

The closed-loop system is stable with this control law, because the following holds:

$$
\frac{d}{d t} x^{T} S_{f} x=-2 x^{T} S_{f} G G^{T} S_{f} x \leq 0
$$

However, asymptotic stability of the origin is not guaranteed, since $G^{T} S_{f} x=0$ holds for $x=\left(x_{1}, 0,0\right)$ with $x_{1}$ nonzero. The trajectory of the closed-loop system is shown in Fig. 3, and time histories of some variables are presented in Fig. 4. The model is drawn every 2 seconds in Fig. 3. The Euclidean norm of the function $F,\|F\|$, is plotted in the time histories in order to evaluate the accuracy of the real-time optimization. The optimal solution is exactly obtained and $\|F\| \equiv 0$ if the terminal time is zero, as is shown in Fig. 4. However, the closed-loop response is not satisfactory because of the large steady-state deviation from the origin. This result shows that optimization based on the prediction is necessary in the feedback control of the present system that has less control inputs than the states. It is also seen in Fig. 4 that time histories of $x_{1}$ and $x_{2}$ do not agree well between the experimental and simulation results, while there is no error in the optimality condition. It may be concluded from this observation that there is modeling error in the dynamics of the experimental model.

In order to demonstrate the necessity for stabilization in the computational algorithm, Fig. 5 shows the closed-loop responses with $\zeta=0$, though the terminal time is increased to $T_{f}=1[\mathrm{sec}]$. The Euler-Lagrange equations and the

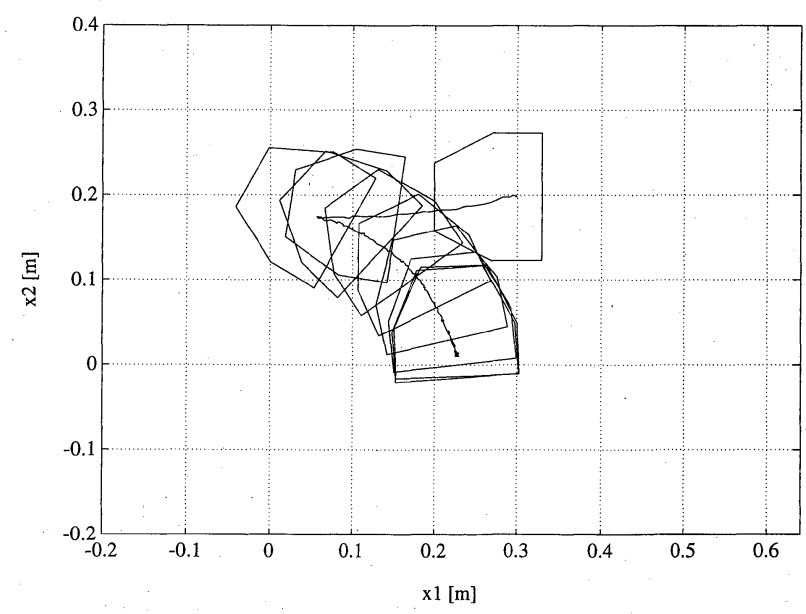

Fig. 3 Trajectory of the closed-loop system (experiment): $T_{f}=0$ 
variational equation are discretized with the time step of $0.2[\mathrm{sec}]$. The Adams method is used for numerical integration along the $\tau$-axis starting with the Runge-KuttaGill method. The maximal error in $F$ is about 0.07 , and the attitude angle $x_{3}$ diverges, which shows that the pres-
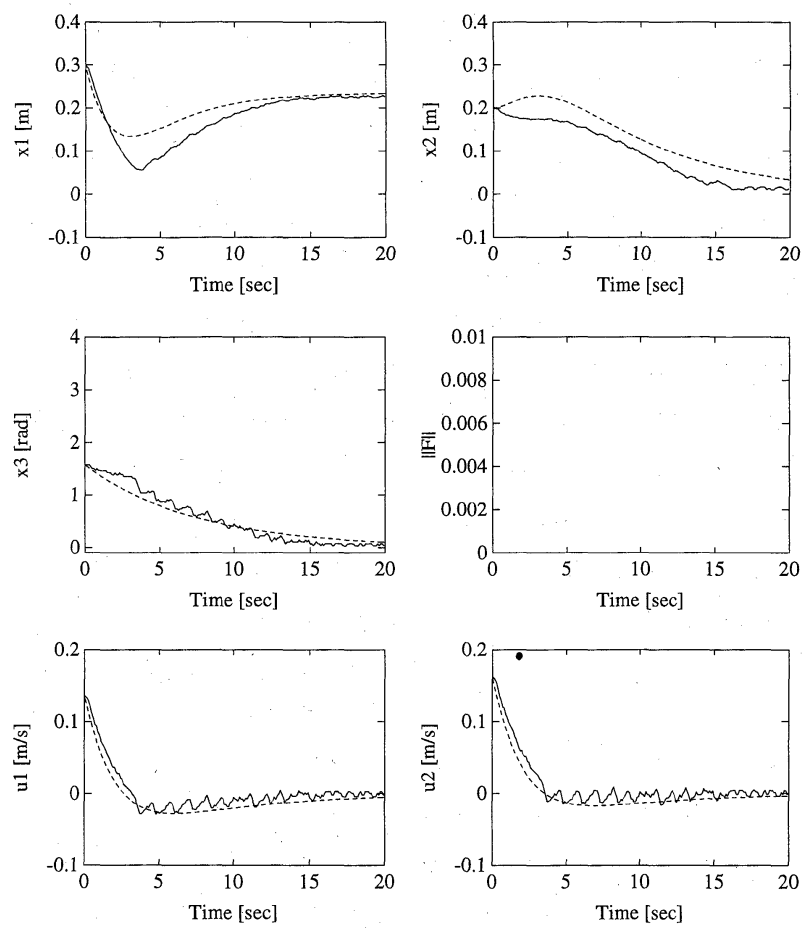

Fig. 4 Time histories of the closed-loop responses (solid line : experiment, broken line : simulation) : $T_{f}=0$.
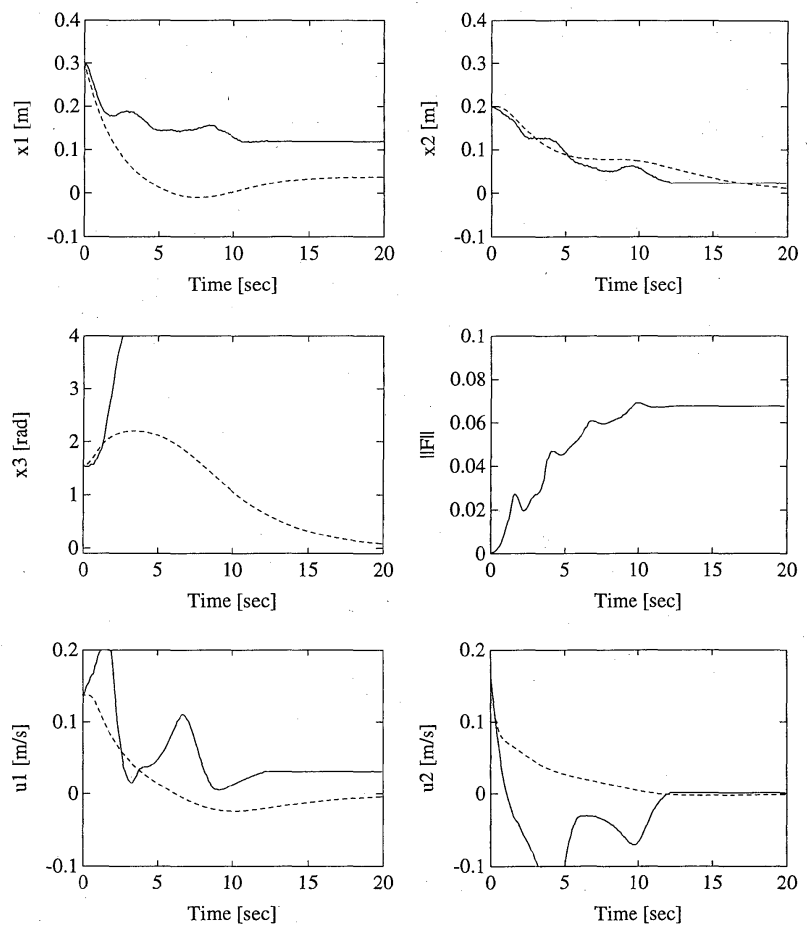

Fig. 5 Time histories of the closed-loop responses (solid line: experiment, broken line: simulation): $T_{f}=1[\mathrm{sec}]$, $\zeta=0$. ent algorithm does not work satisfactorily without the stabilizing term.

In contrast to the above results, the model is controlled successfully with the less steady-state deviation in the case of Figs. 6 and 7 where $T_{f}$ and $\zeta$ are set as $T_{f}=1[\mathrm{sec}]$ and $\zeta=5$, respectively. The steady-state deviation in the state can be further reduced by increasing the terminal weights or the terminal time, as is analyzed later. In spite of the modeling error, the maximal error in $F$ is in the order of $10^{-3}$. It may be concluded that the optimality condition is satisfied in sufficient accuracy throughout the

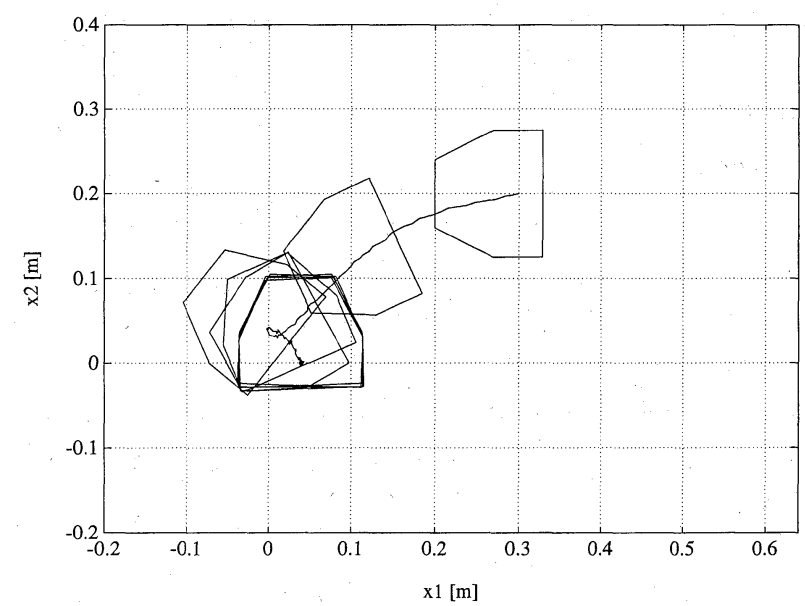

Fig. 6 Trajectory of the closed-loop system (experiment): $T_{f}=1[\mathrm{sec}], \zeta=5$
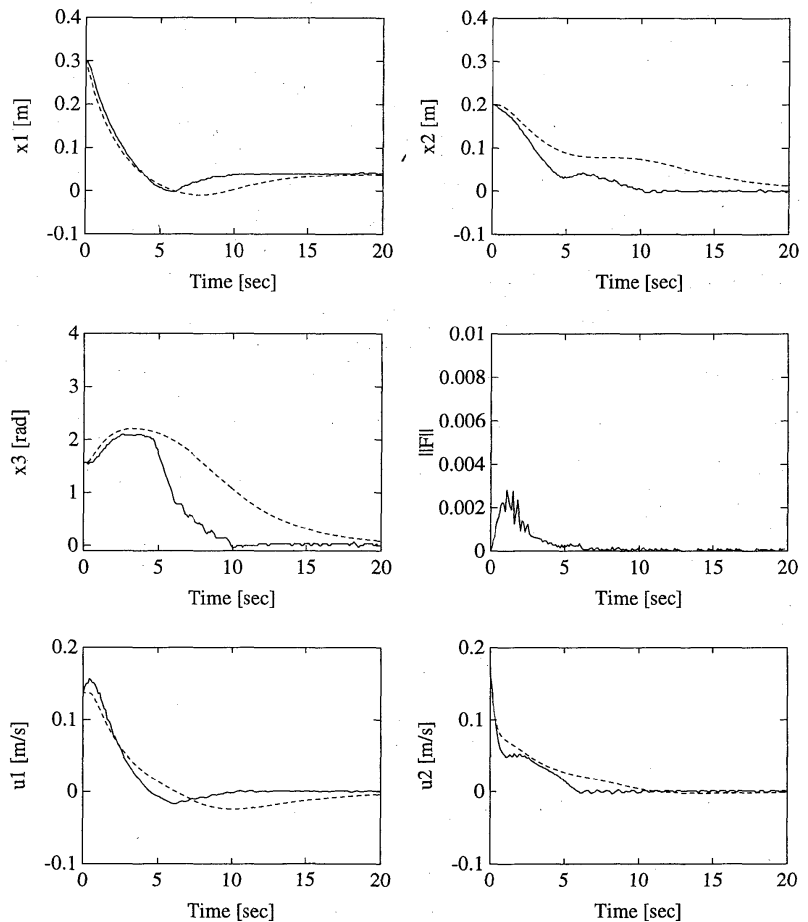

Fig. 7 Time histories of the closed-loop responses (solid line: experiment, broken line: simulation): $T_{f}=1[\mathrm{sec}]$, $\zeta=5$. 
control process.

\section{4 Analysis of Equilibrium Points}

Above experimental and simulation results show that the origin is not the only stable equilibrium point under the proposed control law. For any terminal time $T_{f}$, an equilibrium point of the closed-loop system is expressed as :

$$
x_{e}=\left[x_{1}, 0,0\right]^{T}, \lambda_{e}=\left[s_{1} x_{1}, 0,0\right]^{T}
$$

where $x_{1}$ is arbitrary. Denoting perturbation of $x$, and $\lambda$ around an equilibrium point by $\Delta x$ and $\Delta \lambda$, respectively, dynamics of $\Delta x$ is expressed as:

$$
\frac{d}{d t} \Delta x=-\frac{1}{2}\left[\begin{array}{c}
0 \\
-s_{1} x_{1} \Delta x_{3}+\Delta \lambda_{2} \\
\Delta \lambda_{3} / L^{2}
\end{array}\right]
$$

The perturbation $\Delta \lambda$ is expressed as $\Delta \lambda=S\left(x_{e}, \lambda_{e}, T_{f}\right) \Delta x$ with $S\left(x_{e}, \lambda_{e}, T_{f}\right)$ defined by the coefficient matrix of $d x /$ $d t$ in Eq. (25), and $d \Delta x_{1} / d t$ is zero identically. Therefore the equilibrium point is stable if the following linear system is stable:

$$
\frac{d}{d t}\left[\begin{array}{c}
\Delta x_{2} \\
\Delta x_{3}
\end{array}\right]=\frac{1}{2}\left[\begin{array}{cc}
s_{22} & s_{23}-s_{1} x_{1} \\
s_{23} / L^{2} & s_{33} / L^{2}
\end{array}\right]\left[\begin{array}{c}
\Delta x_{2} \\
\Delta x_{3}
\end{array}\right]
$$

where $s_{i j}$ denotes the $(i, j)$ element of the matrix $S\left(x_{e}, \lambda_{e}\right.$, $\left.T_{f}\right)$. The stability bound $x_{1 \text { sb }}$ on the positive $x_{1}$-axis is given as the minimum of $x_{1}>0$ for which the real part of at least one eigenvalue of the above linear system is nonnegative. It is apparent from the symmetry in the system that $-x_{1 s b}$ is the stability bound on the negative $x_{1}$-axis. The stable equilibrium points of the closed-loop system are given by a set $S_{s e}=\left\{\left(x_{1}, 0,0\right) \mid-x_{1 \mathrm{sb}}<x_{1}<x_{1 \mathrm{sb}}\right\}$. The width of the set $S_{s e}$ is given by $2 x_{1 \text { sb. The stability }}$ bound $x_{1 \mathrm{sb}}$ is plotted in Fig. 8 with the terminal time $T_{f}$ and the terminal penalty $s_{3}$ varied. In Fig. 9, the terminal weights $s_{1}, s_{2}$ and $s_{3}$ are varied. Every point on the $x_{1}$-axis is a stable equilibrium point for the zero terminal time. As shown by Figs. 8 and 9, the width of the set $S_{s e}$ can be reduced by increasing $T_{f}$, by increasing $s_{1}$ and $s_{2}$, or by decreasing $s_{3}$. Although the maximal steady-state deviation in $x_{1}$ can be assigned by choosing those parameters appropriately, there is a certain limitation on choosing $T_{f}$, $s_{1}, s_{2}$ and $s_{3}$ in practice. The possible value of $T_{f}$ is bounded by the computational capability needed to achieve a sufficiently short sample interval. Excessively large $s_{1}$ and $s_{2}$ are not allowed, because they cause excessive magnitude in the control input and the discretized control algorithm fails to trace the optimal solution that varies rapidly. The weight $s_{3}$ has to have a sufficiently large value so that the attitude angle is controlled satisfactorily. The free parameters have to be chosen through a trade-off between the control performance and the neces-

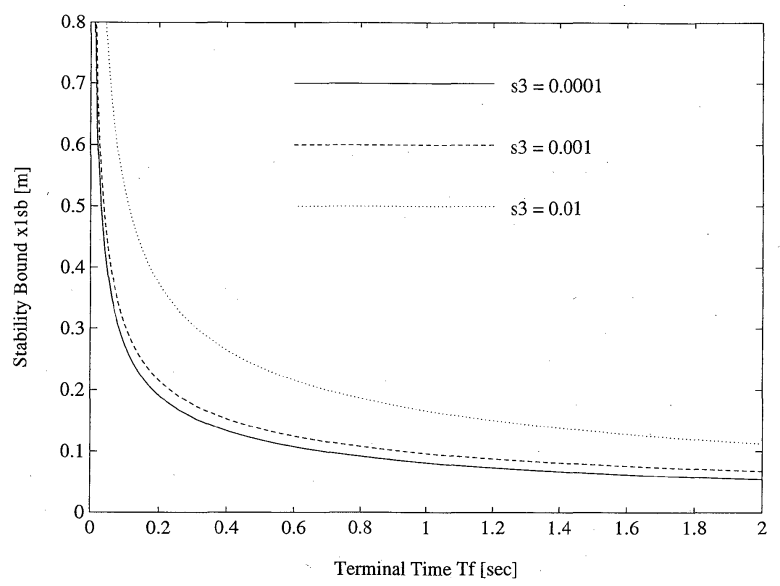

Fig. 8 Stability bound $x_{1 \text { sb }}$ on the positive $x_{1}$-axis versus the terminal time $T_{f}\left(s_{1}=s_{2}=1\right)$

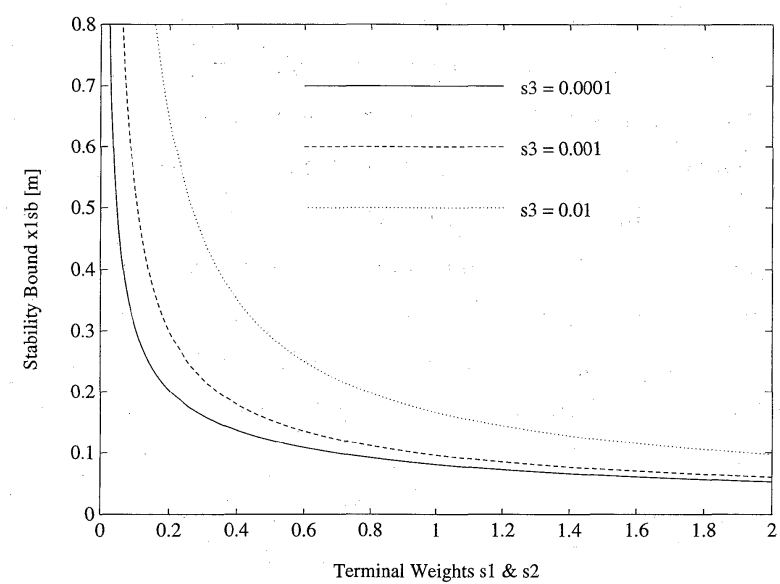

Fig. 9 Stability bound $x_{1 \text { sb }}$ on the positive $x_{1}$-axis versus the terminal weights $s_{1}=s_{2}\left(T_{f}=1[\mathrm{sec}]\right)$

sary computational capability. The multiplier method ${ }^{21)}$ (augmented Lagrangian method) is modified and used in Ref. 22 to reduce the steady-state deviation of the receding horizon control, although the details are omitted in this paper.

\section{Conclusions}

A real-time optimization technique is proposed for the optimal state feedback control of general nonlinear systems. The state feedback control law is determined so that a receding-horizon performance index is minimized. Given a performance index that reflects the control objective appropriately, the receding horizon control achieves the best possible performance even if the controlled system is not controllable nor stabilizable. Since the value of a receding-horizon performance index is always finite when the closed-loop system does not have a finite escape time, the receding horizon control can be designed even for systems such that an infinite-horizon performance index is 
unbounded with any control input.

It is shown that the receding horizon control is equivalent to solving a family of optimal control problems with a varying initial state, and the stabilized continuation method is applied to solve it in the real time. The stabilized continuation results in an ordinary differential equation for the costate. The present solution technique does not involve any approximation nor iterative algorithm in principle. Therefore the present technique generates accurate solutions efficiently, and is quite suitable to real-time optimal feedback control.

The proposed solution technique is applied to a control experiment of a 2 -wheeled mobile robot. The model is a nonlinear nonholonomic system. A quadratic performance index is chosen so as to bring the experimental model to the origin as close as possible, and to set the attitude angle zero. The proposed technique is implemented in a digital computer with the sampling interval of $0.1[\mathrm{sec}]$. The necessity of predicting future responses and effectiveness of the stabilizing term are demonstrated in the control experiment. The experimental model is successfully controlled in the experiment with the terminal time of 1.0 [sec], and the error in the optimality condition is suppressed within the order of $10^{-3}$ throughout the control process. Equilibrium points of the closed-loop system are analyzed, and it is shown that stable equilibrium points belong to a set on the $x_{1}$-axis. Therefore the steady-state deviation in the $x_{1}$-coordinate remains with the present control law. Further application of the proposed real-time optimization technique is expected in future not only for various state feedback problems but also for state estimation problems and output feedback problems.

\section{References}

1) A. E. Bryson Jr. and Y.-C. Ho: Applied Optimal Control, Sec. 2.3, 4.2, and 6.1, Hemisphere, Washington, DC (1975)

2) D. H. Jacobson: Extensions of Linear-Quadratic Control, Optimization and Matrix Theory, Sec. 2.4, Academic Press, London (1977)

3) S. T. Glad: Robustness of Nonlinear State Feedback-A Survey, Automatica, 23-4, 425/435 (1987)

4) C. C. Chen and L. Shaw: On Receding Horizon Feedback Control, Automatica, 18-3, 349/352 (1982)

5) D. Q. Mayne and H. Michalska : Receding Horizon Control of Nonlinear Systems, IEEE Transactions on Automatic Control, 35-7, 814/824 (1990)

6) Y. A Thomas: Linear Quadratic Optimal Estimation and Control with Receding Horizon, Electronics Letters, 11-1, 19/21 (1975)

7) W. H. Kwon and A. E. Pearson: A Modified Quadratic Cost Problem and Feedback Stabilization of a Linear System, IEEE Transactions on Automatic Control, AC-22$5,838 / 842$ (1977)

8) W. H. Kwon, A. N. Bruckstein and T. Kailath: Stabilizing
State-Feedback Design via the Moving Horizon Method, International Journal of Control, 37-3, 631/643 (1983)

9) H. Michalska and D. Q. Mayne: Robust Receding Horizon Control of Constrained Nonlinear Systems, IEEE Transactions on Automatic Control, 38-11, 1623/1633 (1993)

10) C. E. Garcia, D. M. Prett and M. Morari : Model Predictive Control: Theory and Practice-A Survey, Automatica, 25 $-3,335 / 348$ (1989)

11) T. Ohtsuka and H. A. Fujii : Analysis and Approximate Solution Technique of Nonlinear Optimal State Feedback Control, Transactions of the Japan Society for Aeronautical and Space Sciences, 37-118, 248/262 (1995)

12) C. E. Garcia and A. M. Morshedi : Quadratic Programming Solution of Dynamic Matrix Control (QDMC), Chemical Engineering Communications, 46-1/3, 73/87 (1986)

13) D. Marqués and M. Morari : Model Predictive Control of Gas Pipeline Networks, Proceedings of the 1986 American Control Conference, Seattle, WA, 349/354 (1986)

14) J. W. Eaton and J. B. Rawlings: Feedback Control of Chemical Processes Using On-Line Optimization Techniques, Computers \& Chemical Engineering, 14-4/5, 469/ 479 (1990)

15) T. Ohtsuka and H. Fujii : Stabilized Continuation Method for Solving Optimal Control Problems, Journal of Guidance, Control, and Dynamics, 17-5, 950/957 (1994)

16) S. L. Richter and R. A. DeCarlo: Continuation Methods: Theory and Applications, IEEE Transactions on Automatic Control, AC-28-6, 660/665 (1983)

17) L. T. Watson: Numerical Linear Algebra Aspects of Globally Convergent Homotopy Methods, SIAM Review, 28-4, 529/545 (1986)

18) P. T. Kabamba, R. W. Longman and S. Jian-Guo: A Homotopy Approach to the Feedback Stabilization of Linear Systems, Journal of Guidance, Control, and Dynamics, 10-5, 422/432 (1987)

19) H. Nijmeijer and A. J. van der Schaft: Nonlinear Dynamical Control Systems, Chap. 3, Springer-Verlag, New York (1990)

20) R. W. Brockett: Asymptotic Stability and Feedback Stabilization, in Differential Geometric Control Theory, R. W. Brockett, R. S. Milman and H. J. Sussmann (eds.), Birk häuser, Boston, MA, 181/191 (1983)

21) M. R. Hestenes: Multiplier and Gradient Methods, Journal of Optimization Theory and Applications, 4-5, 303/320 (1969)

22) T. Ohtsuka: Control of Distributed Parameter Systems and Nonlinear Systems in Aerospace Engineering, Sec. 6.4, Doctoral Dissertation, Tokyo Metropolitan Institute of Technology, Tokyo, Japan (1994) 


\section{Toshiyuki OHтsuka (Member)}

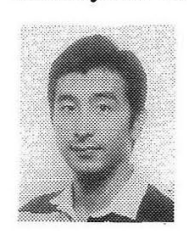

Toshiyuki Ohtsuka was born in Tokyo in 1967. He received the B. Eng., M. Eng., and Dr. Eng. degrees in Aerospace Engineering in 1990, 1992, and 1995 respectively, from the Tokyo Metropolian Institute of Technology. He is currently an Assistant Professor in the Institute of Engineering Mechanics at the University of Tsukuba. His research interests include control theory with application to aerospace engineering. He received the Young Investigator Award of the Japan Society for Aeronautical and Space Sciences in 1996. He is a member of the Japan Society for Aeronautical and Space Sciences, AIAA, JSME, and IEEE.

\section{Hironori A. FuJII (Member)}

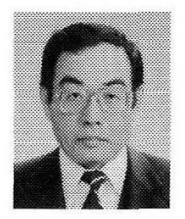

Hironori A. Fujii is a Professor in the Department of Aerospace Engineering at the Tokyo Metropolitan Institute of Technology. He earned his Dr. Eng. degree in 1975, from Kyoto University. His research interests include dynamics and control of large space structures, and robotics for aerospace application. He is the author of about 100 archival journal publications on the study of dynamics and control of space structures. He received the 1994 Best Paper Award of the Japan Society for Aeronautical and Space Sciences. He is an Associate Fellow of AIAA and the Canadian Aeronautics and Space Institute, and he is a member of the American Astronautical Society, the Japan Society for Aeronautical and Space Sciences, and JSME. 